\title{
Transient freezing of liquids in forced laminar flow inside a parallel plate channel
}

\author{
B. Weigand and H. Beer, Darmstadt, BRD
}

\begin{abstract}
A simple analytical approximative solution was given for calculating the time dependent development of the ice-layers at the cooled walls inside a parallel plate channel. By ignoring the effect of acceleration, resulting from converging ice-layers in the axial direction, an analytical solution for the variation of the ice-layer thickness with time and axial position could be obtained. The approximative solution was checked by numerical calculations and good agreement was found.
\end{abstract}

Die zeitliche Entwicklung der Erstarrungsfronten in einem ebenen, gekühlten Kanal mit laminarer Durchströmung

Zusammenfassung. Es wurde ein analytisches Näherungsverfahren entwickelt, das es ermöglicht, die zeitliche Entwicklung der Erstarrungsfronten im gekühlten, ebenen Kanal zu bestimmen. Die Methode liefert unter Vernachlässigung der Beschleunigungsterme durch die konvergenten Eisschichten eine exakte Lösung der Phasengrenzbeziehung. Das Näherungsverfahren wurde mittels numerischer Berechnungen überprüft und stimmt bis zu Wandunterkühlungsverhältnissen von $B=10$ sehr gut mit der numerischen Lösung überein.

\section{Nomenclature}

$B$

$D$

$f$

Fo

$h$

$k$

$P$

$\operatorname{Pr}$

$r_{s}$

Re Reynolds number based on $h$

$R e_{D} \quad$ Reynolds number based on the hydraulic diameter

$T$ temperature

$T_{F} \quad$ freezing temperature of the liquid

$T_{0} \quad$ constant inlet temperature of the liquid

$t$ time

$u, v \quad$ fluid velocity components

$\vec{u} \quad$ mean axial velocity

$\bar{u}_{0} \quad$ mean axial velocity at the entrance

$x, y$ coordinates

$\delta$ distance from centerline to the liquid-solid interface

$\delta_{s} \quad$ steady state distance from centerline

to the liquid-solid interface

\author{
$\varrho \quad$ density \\ $\tau \quad$ dimensionless time \\ $v \quad$ kinematic viscosity \\ $\xi \quad$ integral coordinate \\ Subscripts \\ $s \quad$ solid \\ $L \quad$ liquid \\ $w$ at the wall \\ 0 at the entrance \\ Superscripts \\ $\sim$ dimensionless quantity
}

\section{Introduction}

Problems of solidification or freezing of liquids inside cold channels have been encountered in numerous engineering applications. Because the freeze shut of systems may lead to a destruction of the equipments (for example freeze shut of water pipes in winter or freezing of molten sodium in a nuclear reactor) it is advisable to prevent blockage. If solidification at the cooled channel walls can not be suppressed, steady-state conditions should be achieved.

Many theoretical and experimental studies have been performed for fluid flow in circular tubes with solidification. An early investigation was reported by Zerkle and Sunderland [1] for the steady-state freezing of laminar flow inside a horizontal tube. Under the assumption of a parabolic axial velocity distribution throughout the total axial region and with an appropriate coordinate transformation, they were able to reduce the problem to the classical Graetz problem without solidification. Özisik and Mulligan [2] used a slug flow approximation for the liquid core to analyze transient solidification in an isothermal circular tube. They applied integral transform to obtain the transient development of the ice-layer inside the tube. Bilenas and Jiji [3] solved the boundary-layer equations applying a finite-difference scheme, but they used a wide-meshed grid for their calcula- 
tion. Chida [4] calculated numerically, under consideration of axial conduction, the steady state ice-layer thickness. A fully developed parabolic axial velocity distribution at the entrance of the cooled section was assumed in [3] and [4]. The combined thermal and hydrodynamic development during solidification in an isothermal tube has been analyzed by Hwang and Sheu [5]. Under the assumption that the axial variation in solid layer thickness was small, they were able to reduce the problem to the combined entry region problem without solidification. The simplifications made in [5] give accurate solutions only for small dimensionless freezing parameters $(B \leq 3)$.

Despite its relevance to many important technological and physical problems, the freezing of liquid flows through a cooled two dimensional channel has scarely been studied, both, analytically and experimentally. An early investigation of this problem was reported by Lee and Zerkle [6]. They assumed the axial velocity distribution to be parabolic throughout the chill region, which was in analogy to [1]. With this assumption, the axial velocity distribution yields

$\frac{u}{\bar{u}}=\frac{3}{2}\left[1-\left(\frac{y}{\delta}\right)^{2}\right] ; \quad \bar{u}=\frac{\bar{u}_{0} h}{\delta}$

With the approximated velocity profile according to Eq. (1) and with an appropriate coordinate transformation, the energy equation could then be reduced to a Graetz problem and the steady-state ice layer was calculated. Cheng and Wong [7] extended the model used in [6] to calculate the influence of an externally imposed convective boundary condition on the ice-layer. An experimental investigation of the effect of freezing of liquid in case of laminar flow between two cooled parallel plates was performed only by Kikuchi et al. [8]. Both plates were maintained at the same temperature, which was lower than the freezing temperature of the working fluid, water. The plate wall temperature was varied from $-2^{\circ} \mathrm{C}$ to $-7^{\circ} \mathrm{C}$. A numerical calculation of the steady state ice-layers for arbitrary velocity profiles at the entrance of the chill section was given in [9]. For the case of a fully developed parabolic velocity distribution at the entrance of the cooled channel, the results of [9] were compared with experimental findings [8] and a generally good agreement was found. Bennon and Incropera [10] studied numerically the influence of free convection effects on the axial distribution of the solid-liquid interface in a cooled channel with laminar flow and a fully developed parabolic entrance velocity profile.

The subject of the following study is the presentation of a simple analytical approximative solution for predicting the time dependent development of the ice-layers at the cooled walls inside a two dimensional channel. The approximative solution is presented for two different velocity profiles at the entrance of the chill region

- slug flow

- fully developed parabolic velocity distribution.

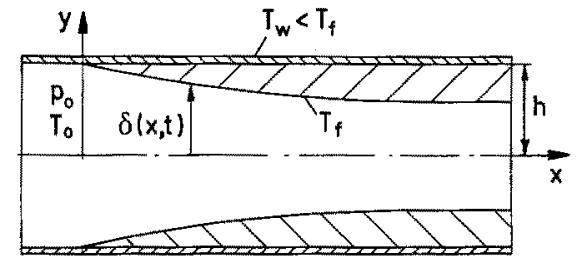

Fig. 1. Physical model and coordinate system

\section{Analysis}

\subsection{Transient liquid solidification}

in the combined hydrodynamic and thermal entrance region

Figure 1 shows the geometrical configuration and the coordinate system for a planar symmetric channel. The laminar fluid flow enters the cooled section at $x=0$ with a slug flow velocity profile and with constant temperature $T_{0}$. In the cooled section $(x>0)$ the wall temperature is maintained at a constant value $T_{w}$ which is lower than the freezing temperature $T_{f}$ of the fluid. The frozen layers are formed on both walls as the fluid proceeds through the channel. By assuming an incompressible, Newtonian fluid with constant fluid properties, the conservation equations for the fluid may be written as follows:

$\frac{\partial u}{\partial x}+\frac{\partial v}{\partial y}=0$

$u \frac{\partial u}{\partial x}+v \frac{\partial u}{\partial y}=-\frac{1}{\varrho} \frac{\partial p}{\partial x}+v \frac{\partial^{2} u}{\partial y^{2}}$

$u \frac{\partial T_{L}}{\partial x}+v \frac{\partial T_{L}}{\partial y}=a \frac{\partial^{2} T_{L}}{\partial y^{2}}$

By deriving Eqs. (3) and (4) the usual boundary-layer assumptions were made, which are a common treatment of the conservation equations for channel flow [3,11, 12]. Further, quasi-steady conditions are assumed. This is justified because the typical time scale of the fluid motion $\left(\delta^{2} / v\right)$ is much shorter than the typical time scale of the freezing process $\left(r_{s} \delta^{2} \varrho / k_{F}\left(T_{0}-T_{F}\right)\right)$.

After neglecting the momentum equation in $y$-direction, the presence of $\partial p / \partial x$ in Eq. (3) introduces an additional unknown to the system given by Eqs. (2)-(4). Thus another equation is needed and is provided by the conservation of mass in integral form

$\bar{u}_{0} h=\int_{0}^{\delta} u d y-\frac{\varrho_{s}-\varrho_{L}}{\varrho_{L}} \int_{0}^{x} \frac{\partial \delta}{\partial t} \mathrm{~d} x$

which presumes a constant flow rate at the entrance of the chill region. $\bar{u}_{0}$ is the mean axial velocity at $x=0$. The boundary conditions belonging to Eqs. (1)-(4) are:

$x=0: \quad p=p_{0}, \quad u=\vec{u}_{0}, \quad v=0, \quad T_{L}=T_{0}$

$y=0: \quad \frac{\partial T_{L}}{\partial y}=\frac{\partial u}{\partial y}=v=0$ 


$$
\begin{aligned}
y=\delta: & v=\frac{\varrho_{L}-\varrho_{s}}{\varrho_{L}} \frac{\partial \delta}{\partial t}\left[1+\left(\frac{\partial \delta}{\partial x}\right)^{2}\right]^{-1}, \\
u & =-v \frac{\partial \delta}{\partial x}, \quad T_{L}=T_{F}
\end{aligned}
$$

Introducing the following dimensionless quantities into Eqs. (2) $-(5)$

$$
\begin{aligned}
& \tilde{y}=\frac{y}{\delta} ; \quad \tilde{x}=\frac{x}{h} \frac{1}{R e_{h}} ; \quad R e_{h}=\frac{\bar{u}_{0} h}{v} ; \quad \tilde{\delta}=\frac{\delta}{h} ; \quad \tilde{\varrho}=\frac{\varrho_{L}-\varrho_{s}}{\varrho_{L}} \\
& \tilde{u}=\frac{u}{\bar{u}_{0}} \frac{\delta}{h} ; \quad \tilde{v}=\frac{v}{\bar{u}_{0}} R e_{h} ; \quad \tilde{p}=\frac{p}{\varrho \bar{u}_{0}^{2}} ; \quad \theta=\frac{T-T_{F}}{T_{0}-T_{F}} \\
& \tau=\text { Fo Ste } \\
& \text { Fo }=\frac{t a_{s}}{h^{2}} ; \quad \text { Ste }=\frac{c_{s}\left(T_{F}-T_{w}\right)}{r_{s}}
\end{aligned}
$$

results in the ensueing set of equations

$$
\begin{aligned}
& \frac{\partial \tilde{u}}{\partial \tilde{x}}-\frac{1}{\tilde{\delta}} \frac{\partial \tilde{\delta}}{\partial \tilde{x}}\left[\tilde{u}+\tilde{y} \frac{\partial \tilde{u}}{\partial \tilde{y}}\right]+\frac{\partial \tilde{v}}{\partial \tilde{y}}=0 \\
& \tilde{u} \frac{\partial \tilde{u}}{\partial \tilde{x}}-\frac{\tilde{u}}{\tilde{\delta}} \frac{\partial \tilde{\delta}}{\partial \tilde{x}}\left[\tilde{u}+\tilde{y} \frac{\partial \tilde{u}}{\partial \tilde{y}}\right]+\tilde{v} \frac{\partial \tilde{u}}{\partial \tilde{y}}=-\tilde{\delta}^{2} \frac{d \tilde{p}}{d \tilde{x}}+\frac{1}{\tilde{\delta}} \frac{\partial^{2} \tilde{u}}{\partial \tilde{y}^{2}} \\
& \tilde{u} \frac{\partial \theta}{\partial \tilde{x}}+\tilde{v} \frac{\partial \theta}{\partial \tilde{y}}-\tilde{u} \frac{\tilde{y}}{\tilde{\delta}} \frac{\partial \tilde{\delta}}{\partial \tilde{x}} \frac{\partial \theta}{\partial \tilde{y}}=\frac{1}{\operatorname{Pr}} \frac{1}{\tilde{\delta}} \frac{\partial^{2} \theta}{\partial \tilde{y}^{2}} \\
& 1=\int_{0}^{1} \tilde{u} d \tilde{y}+\tilde{\varrho} \frac{S t e}{P r} \int_{0}^{\tilde{x}} \frac{\partial \tilde{\delta}}{\partial \tau} d \hat{x}
\end{aligned}
$$

Applying the following coordinate transform

$$
\xi=\int_{0}^{\tilde{x}} \frac{d \hat{x}}{\widetilde{\delta}}
$$

to the conservation equations and inserting a modified velocity component

$\hat{v}=\tilde{\delta} \tilde{v}$

into Eqs. (8)-(10), leads to

$\frac{\partial \tilde{u}}{\partial \tilde{\xi}}-\frac{1}{\tilde{\delta}} \frac{\partial \tilde{\delta}}{\partial \tilde{\xi}}\left[\tilde{u}+\tilde{y} \frac{\partial \tilde{u}}{\partial \tilde{y}}\right]+\frac{\partial \hat{v}}{\partial \tilde{y}}=0$

$\tilde{u} \frac{\partial \tilde{u}}{\partial \xi}-\frac{\tilde{u}}{\tilde{\delta}} \frac{\partial \tilde{\delta}}{\partial \xi}\left[\tilde{u}+\tilde{y} \frac{\partial \tilde{u}}{\partial \tilde{y}}\right]+\hat{v} \frac{\partial \tilde{u}}{\partial \tilde{y}}=-\tilde{\delta}^{2} \frac{d \tilde{p}}{d \xi}+\frac{\partial^{2} \tilde{u}}{\partial \tilde{y}^{2}}$

$\tilde{u} \frac{\partial \theta}{\partial \xi}+\hat{v} \frac{\partial \theta}{\partial \tilde{y}}-\frac{1}{\tilde{\delta}} \frac{\partial \tilde{\delta}}{\partial \xi} \tilde{u} \tilde{y} \frac{\partial \theta}{\partial \tilde{y}}=\frac{1}{\operatorname{Pr}} \frac{\partial^{2} \theta}{\partial \tilde{y}^{2}}$

The boundary conditions are given by

$\tilde{u}(\tilde{y}, 0)=\theta(\tilde{y}, 0)=1 ; \quad \hat{v}(\tilde{y}, 0)=0$

$\hat{v}(1, \xi)=\widetilde{\delta} \frac{S t e}{P r} \tilde{\varrho} \frac{\partial \widetilde{\delta}}{\partial \tau}\left[1+\left(\frac{1}{R e_{h}} \frac{1}{\widetilde{\delta}} \frac{\partial \widetilde{\delta}}{\partial \xi}\right)^{2}\right]^{-1} ;$

$\tilde{u}(1, \xi)=-\frac{\hat{v}}{R e_{h}^{2}} \frac{\partial \widetilde{\delta}}{\partial \xi}$ $\theta(1, \xi)=0$

$\frac{\partial \tilde{u}}{\partial \tilde{y}}(\tilde{y}=0)=\frac{\partial \theta}{\partial \tilde{y}}(\tilde{y}=0)=\hat{v}(0, \xi)=0$

In Eqs. (13)-(16) appear several terms which are of the order $(1 / \tilde{\delta} \partial \tilde{\delta} / \partial \xi)$. They represent the effect of acceleration due to the converging ice-layers. For moderate values of the wall cooling parameter $B$, this terms can be neglected with good accuracy $[5,9]$.

The second integral on the right side of Eq. (11) represents the mass, which gets lost by freezing on the channel walls. For the solidification of water, this term is very small and will be ignored. Because of a change in density between fluid and solid, there is a resulting velocity at the interface, which is given by Eq. (16). However, the velocity components according to Eq. (16) are also very small and can be neglected with good accuracy. Involving the above given simplifications into the conservation equations, Eqs. (13)-(15), results in

$\frac{\partial \tilde{u}}{\partial \xi}+\frac{\partial \hat{v}}{\partial \tilde{y}}=0$

$\tilde{u} \frac{\partial \tilde{u}}{\partial \xi}+\hat{v} \frac{\partial \tilde{u}}{\partial \tilde{y}}=-\tilde{\delta}^{2} \frac{d \tilde{p}}{d \xi}+\frac{\partial^{2} \tilde{u}}{\partial \tilde{y}^{2}}$

$\tilde{u} \frac{\partial \theta}{\partial \xi}+\hat{v} \frac{\partial \theta}{\partial \tilde{y}}=\frac{1}{\operatorname{Pr}} \frac{\partial^{2} \theta}{\partial \tilde{y}^{2}}$

Equations (17)-(19) can be identified as the governing equations for the problem in the combined hydrodynamic and thermal entrance region of a parallel plate channel with the particularity that there is a coefficient $\tilde{\delta}^{2}$ in front of the pressure term $d \tilde{p} / d \xi$.

In the corresponding equations for liquid solidification in the combined hydrodynamic and thermal entrance region in a circular tube [5], the pressure term adopts the form $\tilde{\delta}^{4} d \tilde{p} / d \tilde{x}$. This shows that the pressure loss in cooled pipe with a frozen layer at the wall increases with growing axial distance $\tilde{x}$ more rapidly than in a parallel plate channel.

Finally, it should be stated, that the approximation solution presented here, is correct only for low values of the wall cooling parameter $B$. The range of $B$ for which the approximative solution is valid, will be checked in the next chapter.

By assuming constant properties in the solid region and negligible axial conduction, the heat conduction equation for the solid-phase reduces to

$\frac{\partial^{2} T_{s}}{\partial y^{2}}=0$

The associate boundary conditions are:

$T_{s}(y=\delta)=T_{F}$

$T_{s}(y=h)=T_{w}$

Integrating Eq. (20) according to the boundary conditions Eq. (21), results in the following expression for the tempera- 
ture distribution in the solid region.

$T_{s}=T_{w} \frac{y-\delta}{h-\delta}+T_{F} \frac{h-y}{h-\delta}$

Equations (17)-(19) and Eq. (22) are coupled by the interface energy equation which takes the following form if terms proportional to $(\partial \delta / \partial x)^{2}$ are neglected.

$\left.k_{s} \frac{\partial T_{s}}{\partial y}\right|_{y=\delta}-\left.k_{L} \frac{\partial T_{L}}{\partial y}\right|_{y=\delta}=\varrho_{s} r_{s} \frac{\partial \delta}{\partial t} ; y=\delta$

Equation (23) elucidates that the heat conducted in the solid plus the heat arising from phase change equals the heat transported from the liquid.

Introducing the dimensionless quantities given by Eq. (7) and with Eq. (22), Eq. (23) results in:

$\frac{\tilde{\delta}}{\delta-1}-\left.\frac{1}{B} \frac{\partial \theta}{\partial \tilde{y}}\right|_{\tilde{y}=1}=\tilde{\delta} \frac{\partial \tilde{\delta}}{\delta \tau} ; \quad \tilde{y}=1$

$B$ denotes the dimensionless freezing parameter

$B=\frac{k_{s}}{k_{L}} \frac{T_{F}-T_{W}}{T_{0}-T_{F}}$

The calculation of the frozen layer can be performed in the following manner. Equations (17)-(19) describe the fluid flow and heat transfer in the combined hydrodynamic and thermal entrance region of a parallel plate channel. The solution of this equations is known in detail in literature $[6,12]$ and, therefore, will not be discussed in detail. After solving the conservation equations, the temperature gradient at the solid-liquid interface is known. Figure 2 shows the temperature gradient $(\partial \theta / \partial \hat{y})_{\hat{y}=1}=f(\xi, \operatorname{Pr})$ as a function of $\xi$ for various values of $\mathrm{Pr}$. The plot elucidates that the gradient decreases very rapidly at low values of $\xi$.

After involving the known temperature gradient at the solid-liquid interface into the unsteady state interface equation, Eq. (24), the distribution of $\tilde{\delta}$ can be obtained. It has to be pointed out, that the resulting Eq. (24) is a nonlinear integro-differential equation, because the derivation of $\tilde{\delta}$

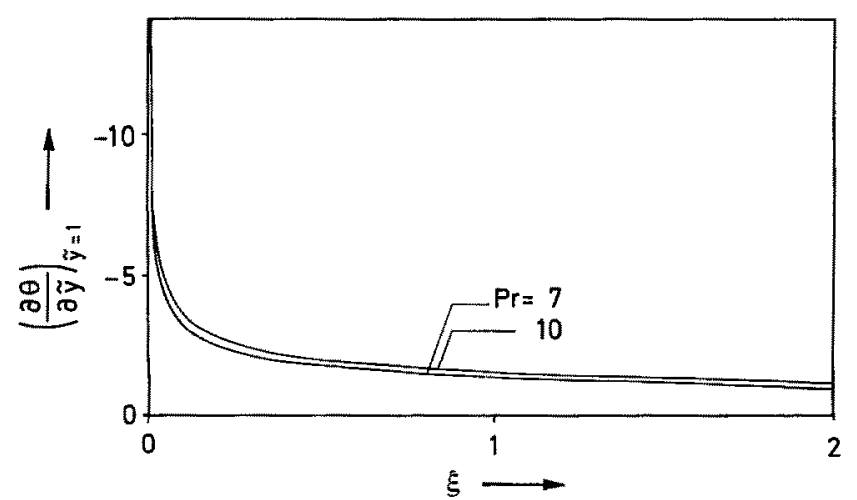

Fig. 2. Temperature gradient at the wall as a function of $\xi$ with $\mathrm{Pr}$ as parameter with time had to be taken at constant $\tilde{x}$. The solution of this integro-differential equation can be obtained only numerically, because of the strong nonlinear character of Eq. (24). However, an approximative solution of Eq. (24) can be derived as follows.

By transforming the axial coordinate in Eq. (24) according to Eq. (12) we get

$\left(\frac{\partial \tilde{\delta}}{\partial \tau}\right)_{\tilde{x}}=\left(\frac{\partial \tilde{\delta}}{\partial \tau}\right)_{\xi}+\left(\frac{\partial \tilde{\delta}}{\partial \xi}\right)_{\tau}\left(\frac{\partial \tilde{\delta}}{\partial \tau}\right)_{\tilde{x}} ; \quad \tilde{y}=1$

In the preceding analysis it was assumed that terms proportional to $1 / \tilde{\delta} \partial \tilde{\delta} / \partial \xi$ are negligible. By employing this assumption in Eq. (26), the second term on the right hand side can be omitted and Eq. (24) reduces to

$\tilde{\delta}\left(\frac{\partial \tilde{\delta}}{\partial \tau}\right)_{\zeta}=\frac{\tilde{\delta}}{\tilde{\delta}-1}-\frac{1}{B} f(\xi, \operatorname{Pr}) ; \quad \tilde{y}=1$

Equation (27) is a first order nonlinear partial differential equation which has to satisfy the following boundary and initial condition for $\tilde{\delta}$.

$\tilde{\delta}(\tau=0, \xi)=1$

$\tilde{\delta}(\tau, \xi=0)=1$

Equation (27) can be integrated analytically. This results in an implicit equation for $\tilde{\delta}$ as a function of the integral coordinate $\xi$ and the dimensionless time $\tau$

$\frac{\tau}{1-\delta_{s}}=\frac{1}{2}\left(\tilde{\delta}^{2}-1\right)+\left(\delta_{s}-1\right)\left[(\tilde{\delta}-1)+\delta_{s} \ln \left(\frac{\tilde{\delta}-\delta_{s}}{1-\delta_{s}}\right)\right]$

where $\delta_{s}$ denotes the steady state distance between channel centerline and solid-liquid interface. $\delta_{s}$ can easily be calculated from Eq. (27), by neglecting the unsteady term on the left hand side. This results in:

$\delta_{s}=\frac{f(\xi, P r)}{f(\xi, P r)-B}$

For a given dimensionless time $\tau_{1}$, Eq. (29) yields $\tilde{\delta}$ as a function of $\xi$. With the transformation relation

$\frac{d \xi}{d \tilde{x}}=\frac{1}{\tilde{\delta}} ; \quad \tilde{x}=0 ; \quad \xi=0 ; \quad \tau=\tau_{1}$

which results from Eq. (12), a relation between $\xi$ and the axial coordinate $\tilde{x}$ can be derived. Therefore, the distribution of $\tilde{\delta}$ with time and with the axial coordinate $\tilde{x}$ is known.

\subsection{Transient liquid solidification \\ for a parabolic entrance velocity profile}

The approximations given in the preceding chapter are also applicable in the case of a fully developed velocity profile at the entrance of the chill region. For convenience we start with the conservation Eqs. (17)-(19), in which the acceleration due to converging ice-layers was neglected, $(\partial \delta / \partial x \rightarrow 0)$. For the case of a parabolic entrance velocity profile the 
governing equations can be written as follows:

$\frac{\partial \tilde{u}}{\partial \xi}+\frac{\partial \hat{v}}{\partial \tilde{y}}=0$

$\tilde{u} \frac{\partial \tilde{u}}{\partial \xi}+\hat{v} \frac{\partial \tilde{u}}{\partial \tilde{y}}=-\tilde{\delta}^{2} \frac{d \tilde{p}}{d \xi}+\frac{\partial^{2} \tilde{u}}{\partial \tilde{y}^{2}}$

$\tilde{u} \frac{\partial \theta}{\partial \xi}+\hat{v} \frac{\partial \theta}{\partial \tilde{y}}=\frac{1}{\operatorname{Pr}} \frac{\partial^{2} \theta}{\partial \tilde{y}^{2}}$

with the conservation of mass in integral form

$1=\int_{0}^{1} \tilde{u} d \tilde{y}$

and the modified boundary conditions

$\tilde{u}(\tilde{y}, 0)=\frac{3}{2}\left(1-\tilde{y}^{2}\right) ; \quad \hat{b}(\tilde{y}, 0)=0 ; \quad \theta(\tilde{y}, 0)=1$

$\theta(1, \xi)=\hat{v}(1, \xi)=\tilde{u}(1, \zeta)=0$

$\frac{\partial \tilde{u}}{\partial \tilde{y}}(\tilde{y}=0)=\frac{\partial \theta}{\partial \tilde{y}}(\tilde{y}=0)=\hat{v}(0, \xi)=0$

The solution of Eqs. (17) and (18), according to the boundary conditions (16a), is given in [16].

$\frac{u}{\bar{u}}=\frac{3}{2}\left[1-\left(\frac{y}{\delta}\right)^{2}\right] ; \quad \bar{u}=\frac{\bar{u}_{0} h}{\delta}$

With the assumed velocity profile according to Eq. (1), Lee and Zerkle [6] were able to solve the energy Eq. (19) with the boundary conditions given by Eq. (16a). After obtaining the temperature gradient at the solid-liquid interface, they calculated an expression for the steady-state distribution of $\widetilde{\delta}$ from the interface energy equation.

By obtaining the profile according to Eq. (1) as a solution of the conservation equation it is assumed, that $\partial \delta / \partial x \rightarrow 0$. Therefore, the transient development of the ice-layers at the cooled channel walls can be calculated with the help of Eq. (29), by inserting the altered temperature gradient in the fluid at the interface into Eq. (29). The temperature gradient at the solid-liquid interface was given by Lee and Zerkle [6] in form of an infinite series

$\left(\frac{\partial \theta}{\partial \tilde{y}}\right)_{\tilde{y}=1}=\sum_{n=0}^{\infty} c_{n} y_{n(1)}^{\prime} \exp \left(-\frac{2}{3} \lambda_{n}^{2} \tilde{\xi}\right)=f(\xi)$

in which $\xi=\xi / P r$. The eigenvalues $\lambda_{n}$ and the coefficients $c_{n} y_{n(1)}^{\prime}$, which appear in Eq. (32), are given in literature [13].

By solving Eqs. (29) - (32) in the same way as described in the preceding chapter, the distance between the channel centerline and the interface is obtained as a function of $\bar{\xi}$ and time $\tau$.

\section{Results}

\subsection{Hydrodynamic- and thermal entrance region}

\subsubsection{Steady state freezing front}

The validity of the assumption that terms of the order $1 / \tilde{\delta} \partial \tilde{\delta} / \partial \xi$ in the conservation equations are negligible will

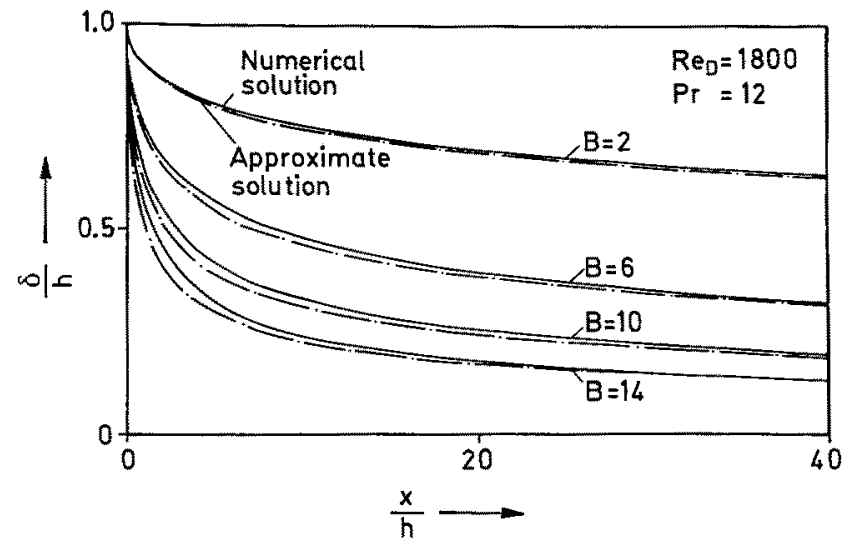

Fig. 3. Steady state ice-layers as a function of $x / h$ with $B$ as parameter in the hydrodynamic- and thermal entrance region

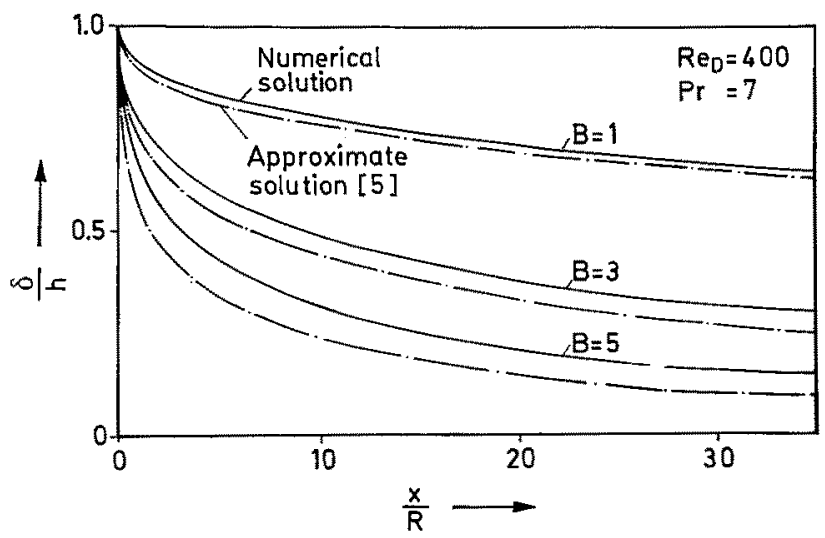

Fig. 4. Steady state ice-layers in a pipe as a function of $x / R$ for various values of $B$

be checked for the steady-state solution. However, this yields an upper limit of error, as $1 / \delta \partial \tilde{\delta} / \partial \xi$ will reach its maximum for steady-state conditions.

In Fig. 3 the steady-state ice-layer thickness is plotted as a function of dimensionless downstream coordinate. It elucidates the deviation of the approximative solution, Eq. (27), from the numerical calculation. The numerical solution was obtained by solving the Eqs. (8) - (11), which include terms of order $1 / \delta \delta \tilde{\delta} / 0 \xi$. For a more detailed information concerning the numerical method see [9].

Figure 3 shows that the approximation, given by Eq. (27), is in very good agreement with the numerical solution for dimensionless freezing parameters $B \leq 10$. In contrast to the applicability of this approximative solution even for high values of $B$, the approximation given by Hwang and Sheu [5] for liquid solidification in the combined hydrodynamic and thermal entrance region of a circular tube, is only valid for small values of $B$. This fact is elucidated by Fig. 4 . It is obvious that the deviation between the numerical solution and the approximative solution given in [5] increases rapidly with growing $B$. Therefore, the approximative solution given by Hwang and Sheu [5] should only be used in a range of the dimensionless freezing parameter $0 \leq B \leq 3$. This results from 
the heat transfer mechanism in a circular pipe, which is quite different from that in a planar channel. This is evidenced by the steady state interface equation. Unlike in circular pipes, where the distance $\delta_{s}$ is proportional to $e^{-B /|\hat{\theta} \theta / \hat{\partial} y| w}, \delta_{s}$ will be proportional to $1 /\left(1+B /\left((\partial \theta / \partial \tilde{y})_{W} \mid\right)\right.$ in planar channels. This shows that the same value of $B$ will lead to a thicker frozen ice layer in a circular pipe than in a planar channel. From a physical point of view this fact is obvious, because the logarithmic temperature profile in the ice-layer inside the cooled tube will give rise to a steeper temperature gradient at the solid liquid interface and, therefore, to a thicker icelayer than in a planar channel, for the same value of $B$. The generally good agreement between theory and experiments, which was mentioned by Hwang and Sheu [5], can be attributed to the low values of the dimensionless freezing parameters $B$, which could be realized in their experiments $(B \approx 1.2)$.

\subsubsection{Transient development of the ice-layer}

The series of Figs. 5-7 illustrates the time dependent development of the freezing fronts at the channel walls for different values of the freezing parameter $B$. In the early stage of the freezing process the ice-layer thickness is approximately constant over the channel length, except for the near entrance region, as can be seen from Fig. 7 for $\tau=0.1$ and $\tau=0.2$. This is evidenced by Eq. (24). If we exclude small values of the axial coordinate $\tilde{x}$, the first term on the left hand side of Eq. (24) dominates the second term. This means that for low values of $\tau$ the growth of the frozen layer at the channel walls is dominated by heat conduction in the thin ice-layer.

The time interval for which heat conduction is the dominant mechanism is related to the dimensionless freezing parameter $B$, which is elucidated by Figs. 5-7 as well as by Eq. (24). The time during which heat conduction in the icelayer is dominating will increase with growing $B$.

Excluding small values of the axial coordinate in Eq. (24) and neglecting the convectiv heat transfered from the fluid to the solid-liquid interface, results in

$\frac{1}{\tilde{\delta}-1}=\frac{\partial \tilde{\delta}}{\partial \tau} ; \quad \tilde{\delta}(\tau=0)=1$

An integration leads to

$\tilde{\delta}=1-\sqrt{2 \tau}$

Equation (34) reveals the above mentioned constant thickness of the freezing front.

Figure 8 shows the axial coordinate $\tilde{x}$ as a function of the transformed integral coordinate $\xi$, defined by Eq. (12), for the same parameters as in Fig. 7. For low values of the dimensionless time $\tau$, there is a linear dependence between $\tilde{x}$ and $\xi$. This is easily shown by inserting Eq. (34) into Eq. (12) and evaluating the integral. With increasing time, the shape of the profile deviates from a straight line. The deviation is more pronounced for low values of $\tilde{x}$. This effect is due to the increasing relevance of heat transfered by convection from

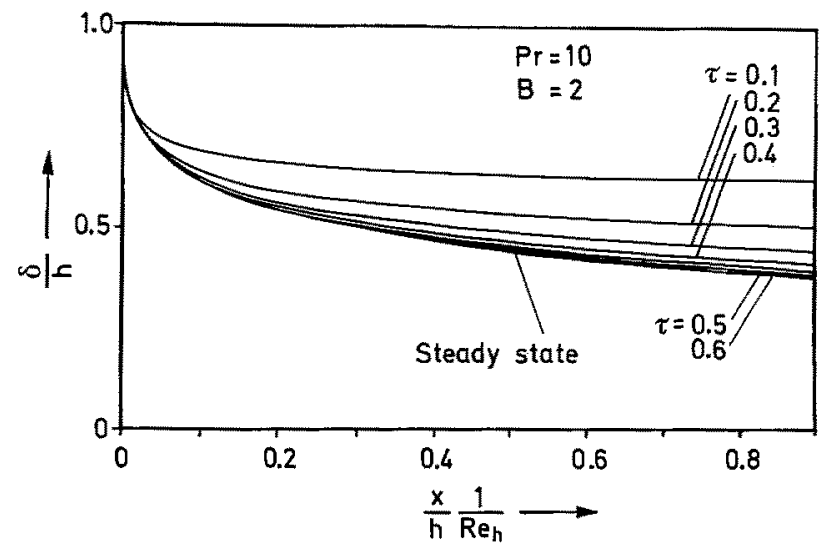

Fig. 5. Transient development of the freezing front as a function of $x / h / R e_{h}$

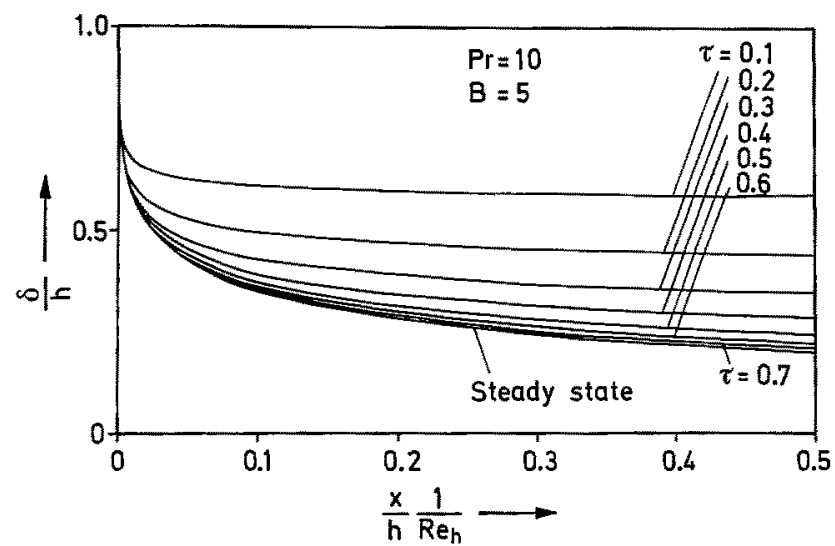

Fig. 6. Transient development of the freezing front as a function of $x / h / R e_{h}$

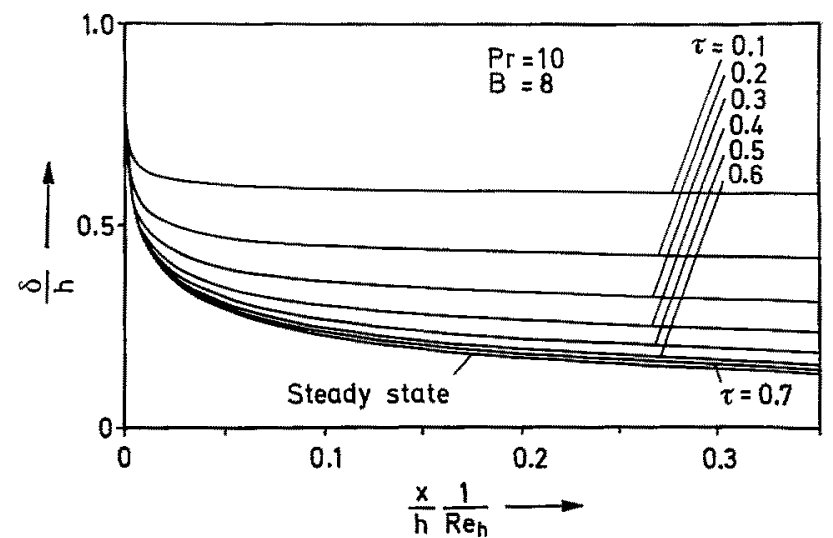

Fig. 7. Transient development of the freezing front as a function of $x / h / R e_{h}$

the fluid to the solid-liquid interface with increasing thickness of the frozen layer.

The second assumption, which led to Eq. (29), was the time derivation of $\tilde{\delta}$ in Eq. (24) for constant $\tilde{x}$ to be replaced by the time derivation of $\widetilde{\delta}$ for constant $\xi$. This requires that the term $(\partial \tilde{\delta} / \partial \xi)_{\tau}(\partial \xi / \partial \tau)_{\tilde{x}}$ in Eq. (26) is negligible. In order 


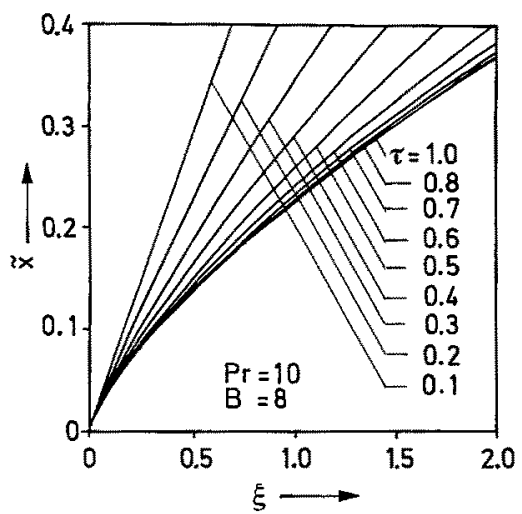

Fig. 8. Variation of the integral coordinate $\xi$ with $\tilde{x}$

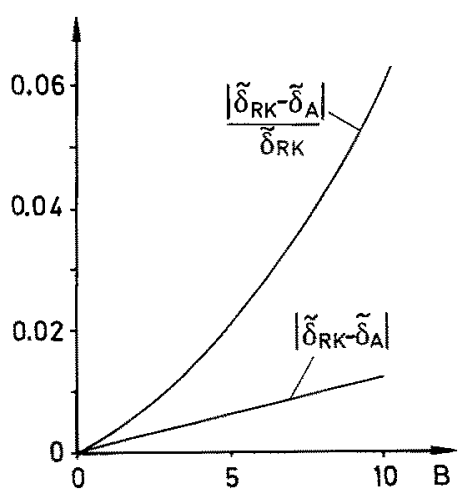

Fig. 9. Relative- and absolute maximum error as a function of $B$

to check this assumption, the integro differential Eq. (24) was solved for various values of $B$ with a fourth order RungeKutta process. Figure 9 shows the maximum relative deviation between the numerical solution $\left(\tilde{\delta}_{R K}\right)$ of Eq. (24) and the approximative solution $\left(\tilde{\delta}_{A}\right)$ according to Eq. (29). It is obvious, that the approximative solution for $B \leq 10$ is in very good agreement with the numerical calculation. This is elucidated by the absolute deviation between $\widetilde{\delta}_{R K}$ and $\widetilde{\delta}_{A}$ which is also plotted in Fig. 9. The absolute deviation for $B \leq 10$ is less than 0.012. The same error bounds were also found in the case of a fully developed velocity profile at the entrance of the chill region.

\subsection{Fully developed entrance profile}

The results obtained in chapter 2.2 are presented in Figs. 10 and 11 . Figure 10 shows a comparison between steady-state freezing fronts according to the approximation given by Lee and Zerkle [6] with a numerical solution of the conservation Eq. (9). It can be seen, that the relative deviation between numerical solution and approximation increases with growing $B$. By comparing Fig, 10 with Fig. 3 it is obvious

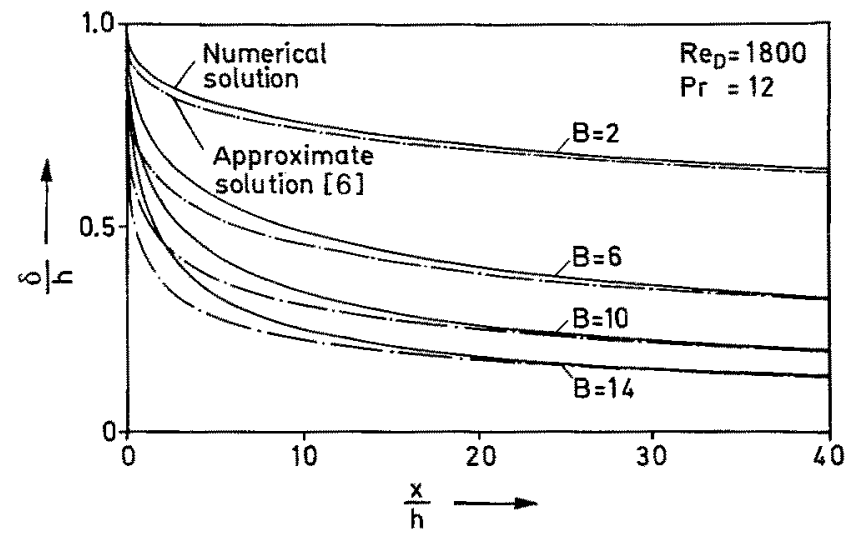

Fig. 10. Steady-state ice-layers as a function of $x / h$ for various $B$ for a fully developed entrance velocity profile

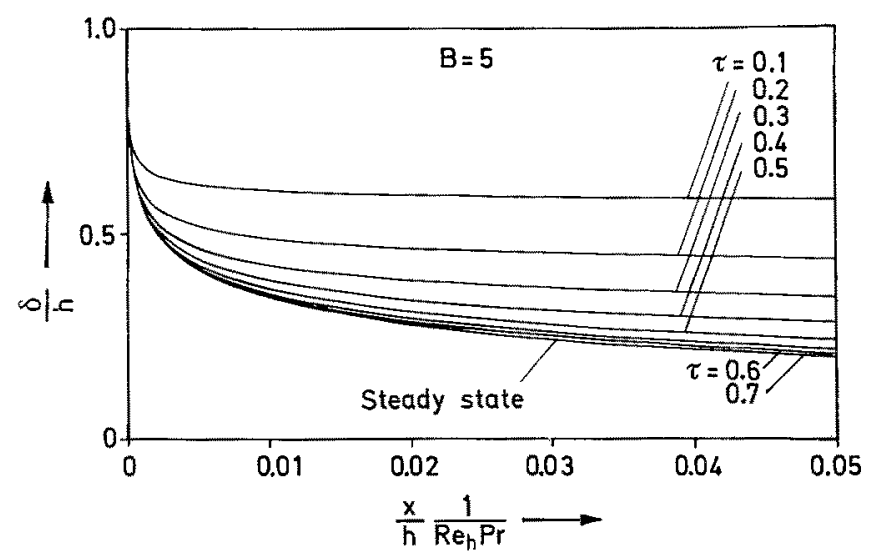

Fig. 11. Transient development of the freezing front as a function of $x / h$ with time as parameter for a fully developed entrance velocity profile

that the deviation between approximative solution and numerical calculation is more pronounced in the case of a fully developed entrance velocity profile. This is due to the velocity profile according to Eq. (1) which is not correct for small values of $x / h$. In this region the actual axial velocity profile is flattened in the near core region and the velocity gradient at the wall becomes much steeper than for a parabolic profile according to Eq. (1). This effect is due to the strong acceleration caused by the converging ice layers in this region. Because of the steeper velocity gradient at the wall the heat transfer is intensified which gives rise to a thinner ice layer in this region. In the case of the hydrodynamic and thermal development (Fig. 3) this effect is covered by the development of the slug flow profile.

Figure 11 shows the transient development of the ice-layer for a dimensionless freezing parameter $B=5$ as a function of the axial coordinate for a fully developed parabolic profile at the entrance of the chill region. The distribution of $\widetilde{\delta}$ in this case is only a function of $\tau$ and $x / h 1 / R e_{h} P r$ and no longer an explicit function of $\mathrm{Pr}$ as in chapter 2.1.

Finally it has to be pointed out that the above given method can not be applied to model the freeze shut of a 
planar channel, because a constant flow rate at the entrance of the chill region is assumed. The freeze shut of a planar channel can be calculated analogously to [14], by requiring a constant pressure difference over the chill region.

\section{Concluding remarks}

A simple analytical approximative solution was given for calculating the transient development of the ice-layers on the cooled walls inside a parallel plate channel. The approximation was checked by a numerical calculations for the steadystate case and good agreement was found up to $B=10$. The model given above can easily be applied to turbulent internal flows by replacing the temperature gradient at the solidliquid interface in Eq. (29).

\section{Acknowledgements}

The support of this work by the Deutsche Forschungsgemeinschaft is gratefully acknowledged.

\section{References}

1. Zerkle, R. D.; Sunderland, J. E.: The effect of liquid solidification in a tube upon laminar-flow heat transfer and pressure drop. J. Heat Transfer 90 (1968) 183-190

2. Özisik, M. N.; Mulligan, J. C.: Transient freezing of liquids in forced flow inside circular tubes. J. Heat Transfer 91 (1969) $385-390$

3. Bilenas, J. A.; Jiji, L. M.: Numerical solution of a nonlinear free boundary problem of axisymmetric fluid flow in tubes with surface solidification. Proc. 4th Int. Heat Transfer Conference 1 Paris. Amsterdam: Elsevier $1970 \mathrm{Cu} 2.1,1-11$

4. Chida, K. Heat Transfer in steady laminar pipe flow with liquid solidification. Heat Transfer: Jap. Res. 81 (1983) 81-94
5. Hwang, G. J; Sheu, J. P.: Liquid solidification in combined hydrodynamic and thermal entrance region of a circular tube. Canadian J. Chem. Eng. 54 (1976) 66-71

6. Lee, D. G.; Zerkle, R. D.: The effect of liquid solidification in a parallel plate channel upon laminar-flow heat transfer and pressure drop. J. Heat Transfer 91 (1969) 583-585

7. Cheng, K. C.; Wong, L. S.: Liquid solidification in a convectively cooled parallel-plate channel. Canadian J. Chem. Eng. 55 (1977) $149-155$

8. Kikuchi, Y; Shigemasa, Y., Ogata, T.: Steady-state freezing of liquids in laminar flow between two parallel plates. J. Nucl. Sci. Technol. 23 (1986) 43-55

9. Weigand, B; Beer, H.: Liquid solidification in a parallel plate channel upon laminar-flow heat transfer: stationary case. Wärme-Stoffübertrag. 26 (1991) 233-240

10. Bennon, W. D.; Incropera, F. P.: Developing laminar mixed convection with solidification in a vertical channel. J. Heat Transfer 110 (1988) 410-415

11. Cebeci, T.; Chang, K. C.: A general method for calculating momentum and heat transfer in laminar and turbulent duct flows. Numerical Heat Transfer 1 (1977) 39-68

12. Cebeci, T.; Bradshaw, P.: Physical and computational aspects of convective heat transfer. New York: Springer 1984

13. Shah, R. K.; London, A. L.: Laminar flow forced convection in ducts. Advances in Heat Transfer. New York: Academic Press 1978

14. Sampson, P.; Gibson, R. D.: A mathematical model of nozzle blockage by freezing. Int. J. Heat Mass Transfer (1981) $231-241$

15. Cebeci, T.; Bradshaw, P.: Momentum transfer in boundary layers. New York: McGraw-Hill 1977

16. Batchelor, G. K.: An introduction to fluid dynamics. Cambridge: Cambridge University Press 1985

Prof. Dr.-Ing. H. Beer

Dipl.-Ing. B. Weigand

Institut für Technische Thermodynamik

Petersenstrabe 30

6100 Darmstadt

Received March 20, 1990 